\title{
SEGUIMIENTO AUTOMATICO DE TORMENTAS CONVECTIVAS POR RADAR
}

\author{
SADIEL NOVO \\ Centro de Física Atmosférica, Instituto de Meteorología \\ Loma de Casablanca, Municipio Regla, Ciudad Habana, Cuba \\ Apartado 17032, Habana 17. C.P. 11700, Cuba \\ Email: sadiel.novo@insmet.cu
}

Recibido Agosto 2006 - Aceptado Marzo 2007

\begin{abstract}
RESUMEN
Se presenta un método automático de identificación y seguimiento de tormentas convectivas por radar, el cual se aplica a dos casos de estudio escogidos como días con reportes de severidad. El método emplea como entrada imágenes CAPPI de reflectividad de radar a $3 \mathrm{~km}$ de altura, consecutivas en el tiempo, obtenidas con el sistema Vesta ver. 5.3.4.5 (Pozas, 2005). Para la identificación se emplea el método de etiquetado de componentes conectados y para el seguimiento se busca la distancia mínima a la próxima posición de la tormenta. Para probar el método se dividieron los datos en tres intervalos de tiempo, de acuerdo a su complejidad observada, y se le aplicó el procedimiento automático a cada caso por separado. En todos los casos se demuestra, por simple inspección visual, la habilidad del método para identificar y seguir de forma correcta las tormentas, siendo mejor para el caso simple y para el de moderada complejidad. Incluso para el caso de gran complejidad el seguimiento se realiza de forma satisfactoria cuando se varía el umbral para la identificación. Por último, se analizan las limitaciones del método y se proponen algunas recomendaciones para el trabajo futuro.
\end{abstract}

\begin{abstract}
AUTOMATIC TRAKING FOR CONVECTIVE STORMS BY RADAR
An automatic method for identifying and tracking convective storms by radar is presented, which is applied to two cases of study chosen as days with reports of severity. The method uses as entry CAPPI images of radar reflectivity $3 \mathrm{~km}$ height, consecutive in time, and obtained with the system Vesta ver. 5.3.4.5 (Pozas, 2005). For the identification the connected component labeling is used, and for tracking we seek for the minimum distance to the next position of the storm. For testing the method, the data were divided in three time intervals according to their observed complexity, and the automatic procedure was tested on every separated case. It is demonstrated, in all the cases by simple visual inspection, the ability of the method to identify and track in a right way the storms, being better for the simple case and for that of moderate complexity. Even for the very complex case, the tracking is done with success when the identification threshold is changed. Finally, the limitations of the method are analyzed and some recommendations for the future work proposed.
\end{abstract}

\section{INTRODUCCIÓN}

Es conocida la gran dificultad de pronosticar la evolución futura de tormentas convectivas, debido fundamentalmente a su rápida evolución y corto tiempo de vida (Houze, 1993). Debido a su alta resolución espacial y temporal, el radar ha sido el dispositivo empleado con más éxito para esta tarea (Wilson et al, 1998). El pronóstico puede hacerse de forma manual mediante el análisis visual de las imágenes de radar consecutivas, pero esta labor puede consumir bastante tiempo y no es factible cuando se necesitan analizar muchos datos. El tiempo consumido por un sistema objetivo de pronóstico no debe exceder aquel que transcurre entre la obtención de dos imágenes consecutivas de radar (usualmente $5 \mathrm{~min}$ ) y el sistema debe ser capaz de extraer tanta información como sea posible 
sobre la estructura de la tormenta y su evolución futura. Uno de los pasos fundamentales para el desarrollo de un sistema objetivo de pronóstico inmediato de tormentas convectivas por radar, es contar con un método capaz de identificar y seguir con precisión aquello que se defina como "tormenta convectiva". Para definir una tormenta convectiva suele emplearse un umbral (o varios) de reflectividad y otro de área o volumen, lo cual elimina datos espúreos o poco importantes y reduce la cantidad de datos a procesar (Dixon \& Wiener, 1993; Johnson et al, 1998; Handwerker, 2002). Para la identificación suele emplearse alguna técnica de reconocimiento de patrones, como es el etiquetado de componentes conectados (Pitas, 2000). Después de identificar cada tormenta en una imagen de radar se procede a extraer sus propiedades (e.g. reflectividad máxima, área que ocupa, centroide, contenido de agua líquida, etc), las cuales se guardan en un fichero donde además se indica la hora correspondiente y algún otro dato importante como fecha, tipo de imagen de radar, distancia máxima de observación del radar, etc. Una vez extraídas las propiedades de cada tormenta para cada instante procesado, se puede realizar el emparentamiento entre las tormentas de cada par de imágenes consecutivas, lo cual constituye el seguimiento temporal. Para realizar un correcto seguimiento en casos de alguna complejidad, debe tenerse en cuenta el surgimiento de nuevas y la disipación de viejas tormentas, además del hecho demostrado (Houze, 1993) de que las tormentas suelen unirse o dividirse con relativa frecuencia, lo cual constituye una de las principales limitantes de cualquier método de seguimiento.

El presente trabajo tiene como objetivo presentar un método de identificación y seguimiento en dos dimensiones de tormentas convectivas por radar, y demostrar su eficacia para tres ejemplos de creciente complejidad. La segunda sección describe los datos de partida empleados y el método seguido, la tercera muestra los resultados del análisis de los tres casos según el grado de complejidad, y la cuarta y última sección analiza las limitaciones del método y propone variantes para mejorarlo.

\section{DATOS Y MÉTODO SEGUIDO}

Los datos básicos empleados corresponden a observaciones del radar MRL-5 $(1=10 \mathrm{~cm})$ automatizado de Camagüey, para una región circular de $180 \mathrm{~km}$ de radio alrededor de la posición del radar. Estas observaciones se tomaron durante los días 21 y 23 de Julio del 2001, en que se reportaron una granizada y un tornado respectivamente, en el marco del experimento EXPONAC-2001, por lo que representan observaciones de gran calidad e interés científico. Una descripción de las condiciones ambientales prevalecientes estos dos días, así como un análisis por radar de estas dos tormentas, puede encontrarse en (Novo, 2003, 2004). El tiempo entre observaciones fue de entre 5 y $10 \mathrm{~min}$. Con dichas observaciones se crearon, empleando el sistema Vesta ver. 5.3.4.5 (Pozas, 2005), mapas CAPPI de reflectividad $(Z)$ a $3 \mathrm{~km}$ de altura con resolución espacial de $1.2 \mathrm{~km}$.

Estas imágenes se emplearon como entrada al método de identificación y seguimiento escrito en Matlab. Primeramente, para cada imagen, se desecharon los valores de reflectividad $(Z)$ por debajo de cierto umbral (típico $10 \mathrm{dBZ}$ ). Luego se procedió a identificar las tormentas, definidas como todo conjunto de pixels unidos por al menos un lado, mediante el método de etiquetado de componentes conectados (Pitas, 2000). Una vez identificadas se desecharon aquellas cuya área fuera menor que cierto umbral (típico 20 pixels). Las tormentas restantes se aproximaron por elipses de iguales momentos centrales de segundo orden que cada una de sus áreas. Estas elipses permitieron, a partir de su centro geométrico, ubicar cada tormenta así como llevarse una idea visual de su forma y orientación.

Para el seguimiento se buscó en la imagen siguiente, entre todas las posiciones calculadas, aquella cuya distancia a la posición de la tormenta que se seguía fuera mínima. Luego se compararon las correspondientes tormentas (padre e hija) en cuanto a su área y posición y si ambas diferencias no superaron ciertos umbrales, se asociaron sus posiciones como consecutivas de la misma tormenta y se trazó una flecha entre ambas para indicar su desplazamiento. Por último, los valores de la posición, área, Z máxima, Z promedio y suma total de $\mathrm{Z}$ para cada tormenta, fueron archivados en un fichero texto.

\section{RESULTADOS}

Caso simple: Como caso de menor complejidad se escogió un intervalo de 4 observaciones (5:01-5:21 pm del 23-7-01) de radar en el cual no hubieran ocurrido uniones ni divisiones en el campo de reflectividad. Sí se permitió el 
surgimiento de nuevas tormentas. La Figura 1a muestra un ejemplo de identificación para dos instantes (5:01 y 5:10 pm) de este intervalo. Las elipses en línea discontinua representan el instante inicial y las de línea continua el final (cuyo mapa CAPPI es el que se muestra superpuesto para comparar). Las pequeñas cruces indican sus centros. Nótese la posición del radar hacia el centro de la figura, indicada por una cruz de mayor tamaño. En estos dos instantes se identificaron respectivamente 5 tormentas. La tormenta al SE y muy cercana al radar representa aquella donde se produjo el tornado reportado este día cerca de las 5:00 pm. Su momento de mayor intensidad ocurrió a las 5:10 pm. La "tormenta" justo al $\mathrm{N}$ del radar recuerda la forma alargada de la Sierra de Cubitas en Camagüey y está ubicada justo en su borde delantero. Como se discutirá más adelante, ésta no es una tormenta real.

La Figura 1b muestra la identificación y el seguimiento durante los 4 instantes del intervalo. Las elipses en línea discontinua y continua significan los instantes inicial y final, respectivamente, mientras las elipses en línea punteada significan los instantes intermedios. Nótese la aparición de una sexta tormenta al SSE del radar. La llamaremos "de reciente formación". Las dos tormentas cercanas entre sí (que llamaremos 1ra y 2da), al NW del radar, sufrieron varias uniones y divisiones fuera del intervalo de tiempo analizado. La tormenta más al S la nombraremos 6ta. La Figura 2 representa una ampliación de cada una de estas tormentas. La escala de reflectividad de radar es la misma que en Figura 1. En (a) se observan las tormentas 1 ra y $2 \mathrm{da}$, cuya componente de movimiento neta es (según las flechas) todo el tiempo hacia el S, sobre todo en la 2da (más pequeña). En (b) se observan la "tormenta" de Sierra de Cubitas y la tormenta tornádica. En el primer caso se observa un aparente movimiento errático, lo que conlleva a pensar en una falsa tormenta producto de la retrodispersión desde un objeto fijo (sin movimiento, en este caso la propia Sierra). En el segundo caso se observa todo el tiempo una componente bien definida hacia el SW y W. En (c) se muestran la tormenta de reciente formación (nótese la ausencia de la elipse inicial discontinua), cuyo movimiento fue en sus dos únicos instantes hacia el SW, y la 6ta y última identificada, cuyo movimiento es hacia el $\mathrm{W}$ franco, aunque no se distingue muy bien producto de la línea costera divisoria.
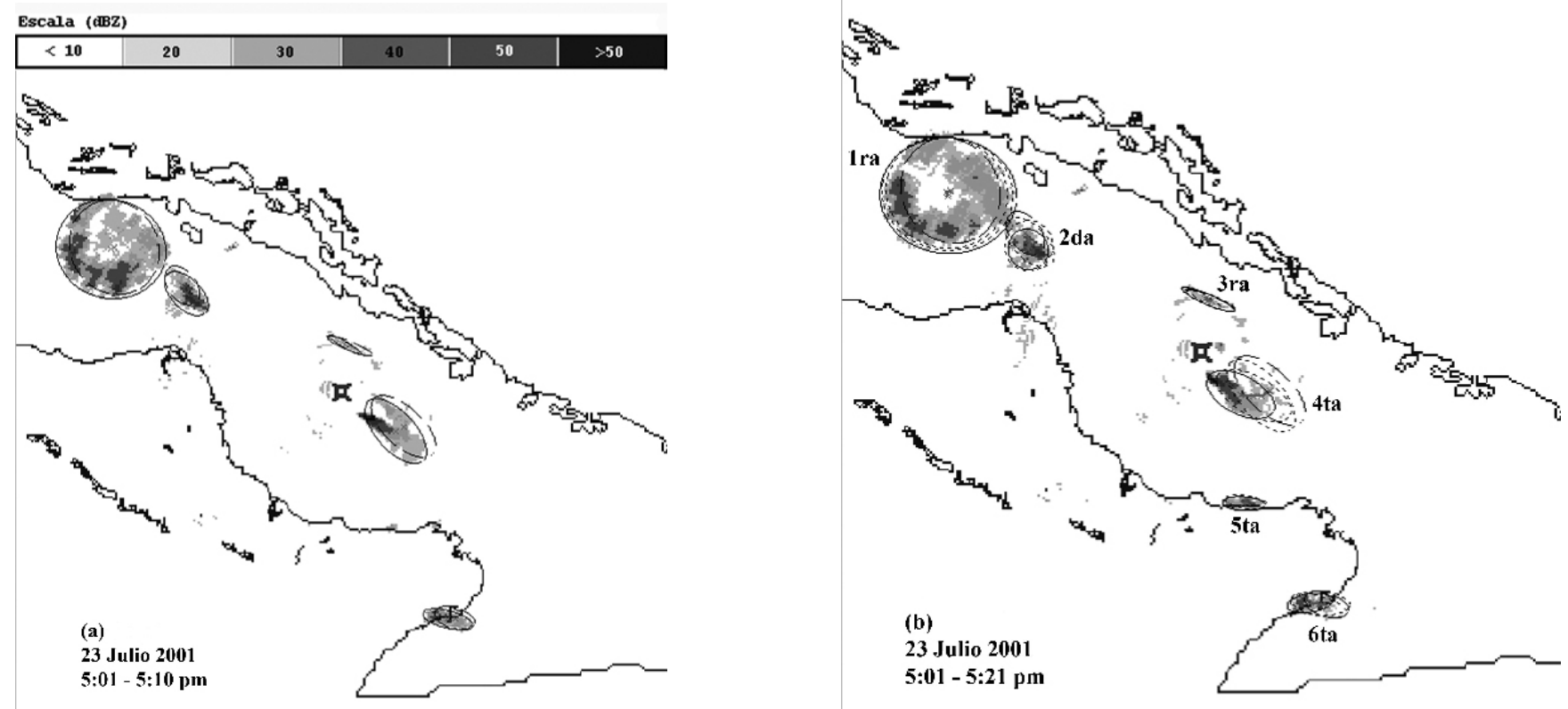

Figura 1 - (a) Identificación para dos instantes, (b) Identificación y seguimiento para el caso simple. 


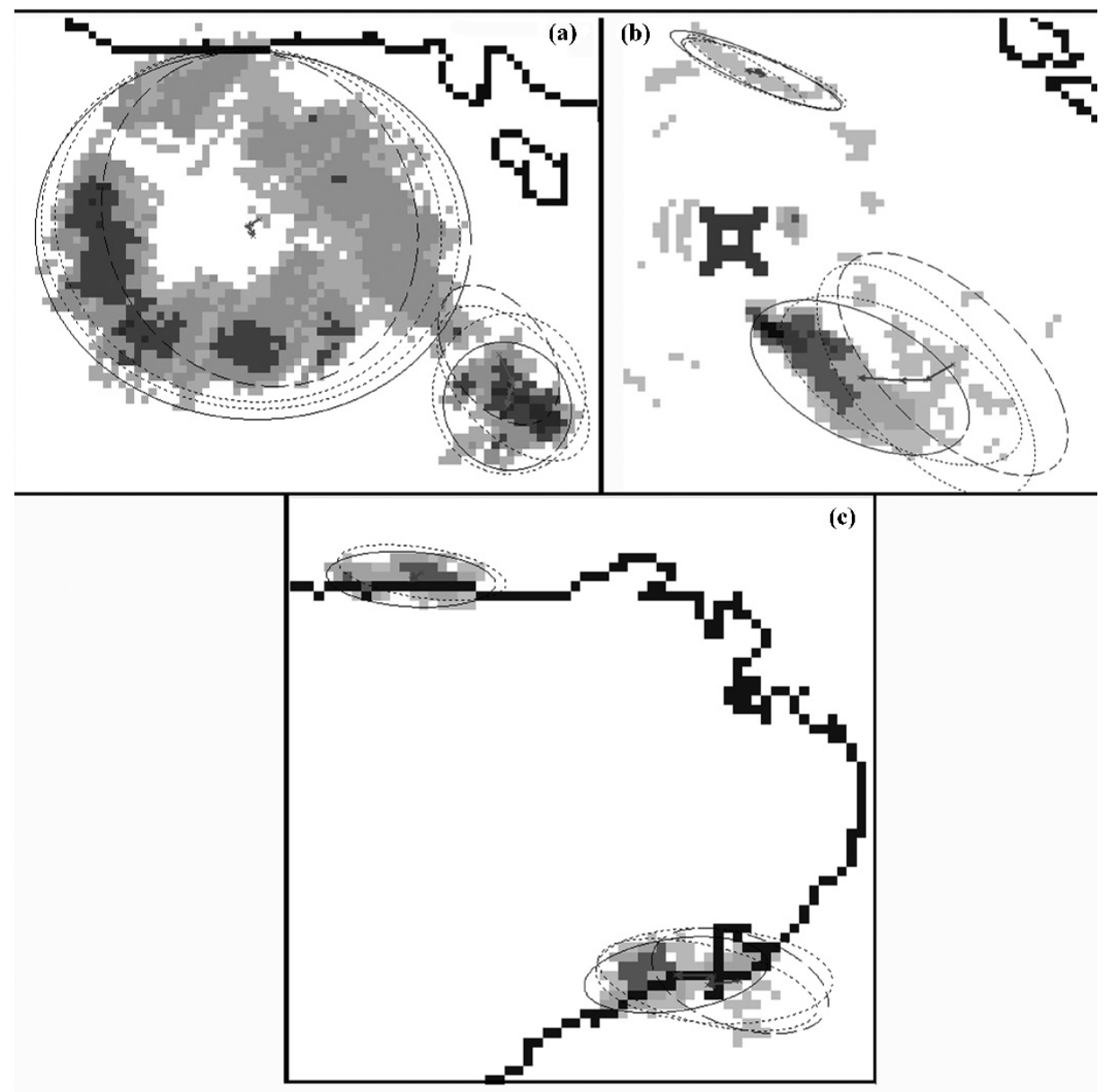

Figura 2 - Ampliación de las tormentas del caso simple.

En todos los casos se observa la clara identificación de las tormentas presentes, así como la adecuación de las elipses para describir de forma general el área ocupada por las tormentas. El área real de cada tormenta se calculó a partir de la cantidad de sus pixels, mientras la $\mathrm{Z}$ máxima, promedio y total de cada tormenta se calculó a partir de sus valores de reflectividad.

Caso complejo: Como caso medianamente complejo se tomaron 8 observaciones del día 23 , entre 4:50 y 5:35 pm, durante las cuales ocurrieron varias fusiones y divisiones entre las tormentas presentes. La Figura 3 muestra la identificación y seguimiento para este intervalo. En el instante final, correspondiente al mapa CAPPI mostrado, se distinguen 7 tormentas (elipses continuas). Nuevamente para la ampliación y análisis dividiremos el mapa en tres zonas: la primera al WNW del radar, la segunda al N y SE cercana al radar, y la tercera al SSW lejana al radar.

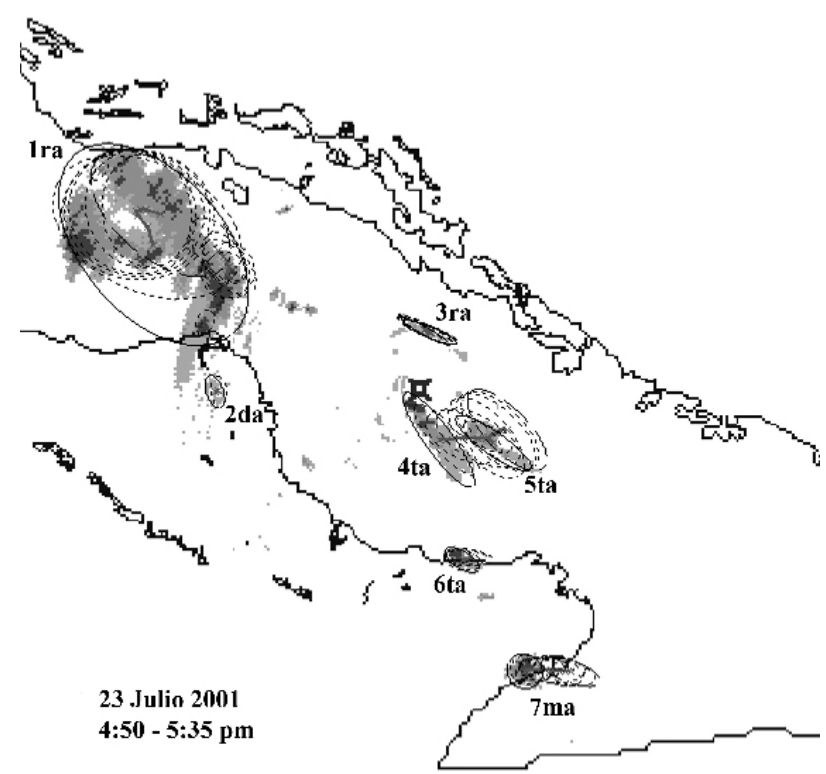

Figura 3 - Identificación y seguimiento para el caso de moderada complejidad. 
La Figura 4 muestra la ampliación de estas zonas. En (a) aparecen 2 tormentas en este instante final, la segunda de ellas de reciente formación a partir del penúltimo instante $(5: 30 \mathrm{pm})$ del intervalo. La primera ha sufrido varios procesos de uniones y divisiones. En el instante inicial aparece como una sola tormenta, indicada por la elipse discontinua. A partir del segundo instante $(4: 55 \mathrm{pm})$ se divide en tres tormentas, comenzando la primera de ellas (la mayor) a moverse al WSW, mientras la segunda en tamaño lo hace al S. En el penúltimo instante estas dos tormentas vuelven a unirse para formar una de mayor tamaño, mientras surgen dos pequeñas al $\mathrm{S}$ de la segunda. Por último, en el instante final una de las tormentas pequeñas se une a la mayor para formar otra de grandes dimensiones, identificada como 1ra tormenta al final del intervalo. El centro de esta tormenta mayor se asocia a la continuación de la tormenta que presentó el mayor tamaño durante todo el intervalo, como es de esperar. El movimiento neto de todo el sistema es hacia el S, como indica su seguimiento detallado.
En la Figura 4(b) se muestra al $\mathrm{N}$ del radar la "tormenta" de Sierra de Cubitas, la cual continúa sin dirección de movimiento definida, apoyando la hipótesis de falsa tormenta. También aparece, en el instante final mostrado, la tormenta tornádica del día 23 dividida en dos. Puede observarse que la división ocurrió en el penúltimo instante $(5: 30 \mathrm{pm})$. La parte principal de la tormenta (nombrada 3ra en Figura 3) continuó su movimiento hacia el W, mientras el pedazo desprendido (4ta en Figura 3) se mueve en los dos instantes finales hacia el SE. Nótese como el método asigna correctamente para el seguimiento la parte principal de la tormenta dividida.

Por último, en la Figura 4(c) aparece la tormenta de "reciente formación" (nombrada 6ta en Figura 3) al N, cuyo movimiento continuó hacia el SW. La 7 ma y última tormenta de este caso se distingue bien al $\mathrm{S}$ y continuó su movimiento al $\mathrm{W}$ franco durante todo el intervalo. Nótese cómo su forma ha cambiado de alargada en los primeros instantes a circular.

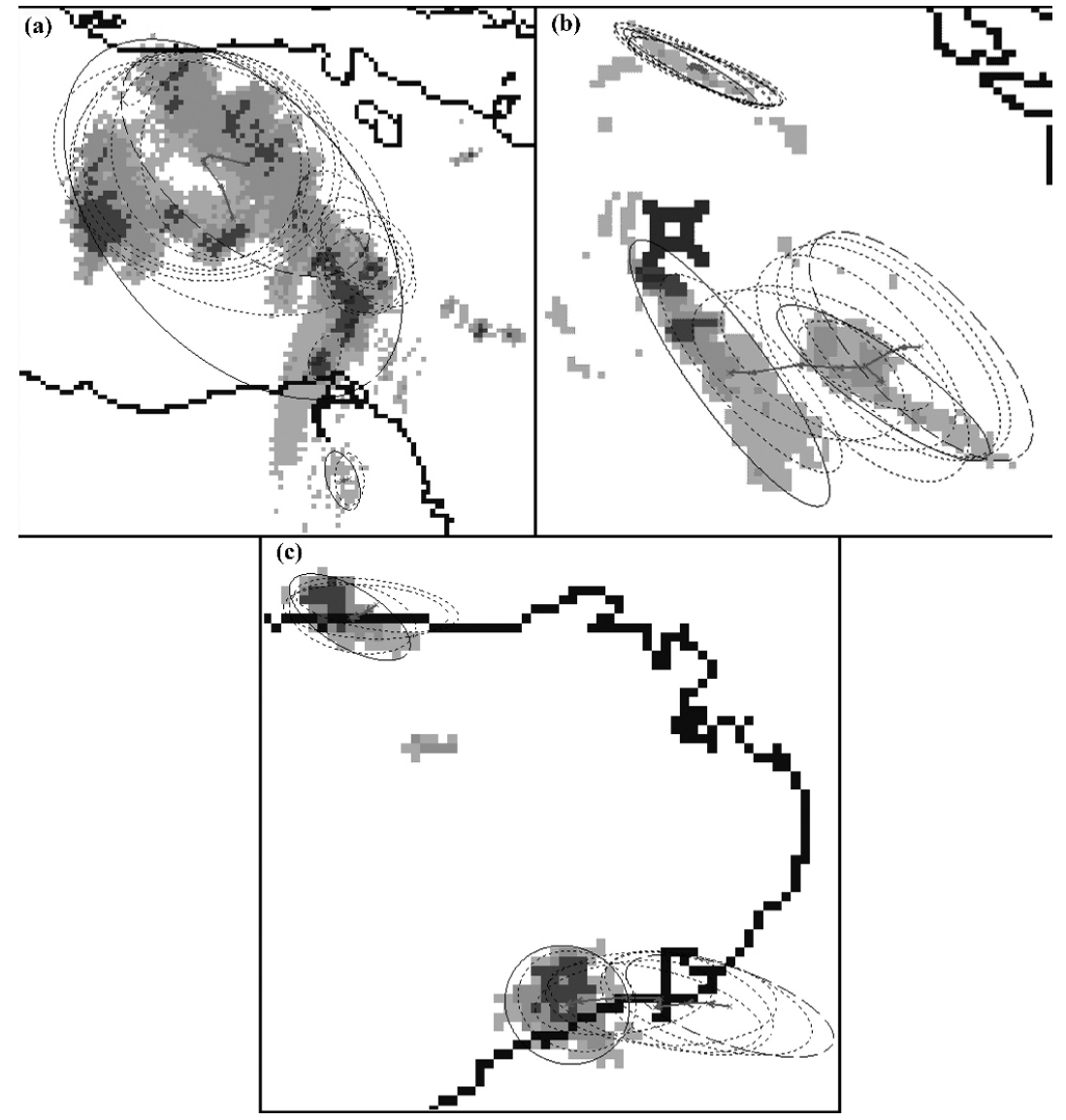

Figura 4 - Ampliación de las tormentas del caso moderadamente complejo. 
Toda la información descrita aparece reflejada tanto en las imágenes anteriores como en cifras numéricas en el fichero texto de salida del programa.

Caso muy complejo: Para este caso se escogió un intervalo de 3 horas (5:30-8:30 pm), correspondiente al surgimiento, desarrollo y casi hasta la disipación de una fuerte tormenta que produjo una granizada en Nuevitas, Camagüey, el día 21 de Julio del 2001. Este caso mostró múltiples uniones y divisiones entre las tormentas presentes. Del estudio preliminar de este día, se decidió emplear un mayor umbral (30 dBZ) de reflectividad para la identificación, pues el alto número de tormentas presentes dificultó mucho el seguimiento de tormentas pequeñas con el anterior umbral de $10 \mathrm{dBZ}$. Este mayor umbral permitió la correcta identificación de tormentas pequeñas embebidas en clusters, por lo que se pudo emplear un menor umbral de área (10 pixels). Para facilitar el análisis visual de las imágenes, dividiremos el intervalo de tiempo en tres partes: $(5: 30-6: 10 \mathrm{pm}, 7$ instantes), (6:10-7:10 pm, 9 instantes) y (7:10-8:30 pm, 13 instantes). La Figura 5 muestra todas las tormentas presentes, en el área de visión del radar, para el último instante del primer intervalo, con la identificación y el seguimiento superpuestos. Nos concentraremos en la región más cercana justo al $\mathrm{E}$ del radar (indicada con un rectángulo), que fue donde se originó la fuerte tormenta que produjo granizos más tarde.

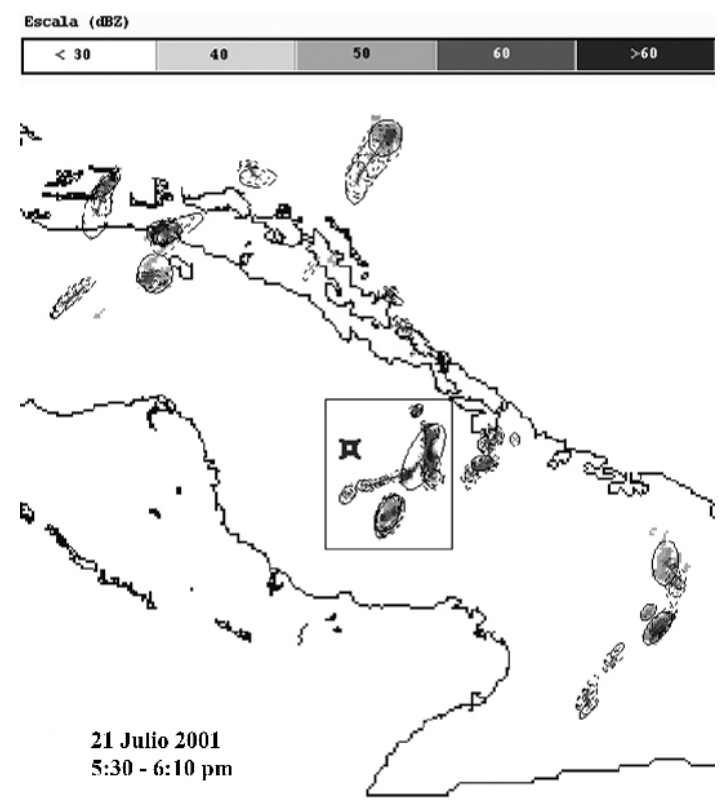

Figura 5 - Identificación y seguimiento para el caso de gran complejidad.
En la Figura 6(a) se ha ampliado la región del rectángulo. Como se ve justo al Este del radar, la mayor tormenta (que es también la más intensa de todas las presentes) se ha formado por la unión de tres tormentas menores que se movieron en diferentes direcciones hacia un mismo punto central; una vino del SW, otra del N y la tercera del S. Las que vinieron de región $\mathrm{S}$ existían desde el comienzo del intervalo (5:30 pm), mientras que la proveniente del $\mathrm{N}$ fue de más reciente formación. Esta gran unión fue la primera de varias de gran magnitud en toda la evolución de esta tormenta.

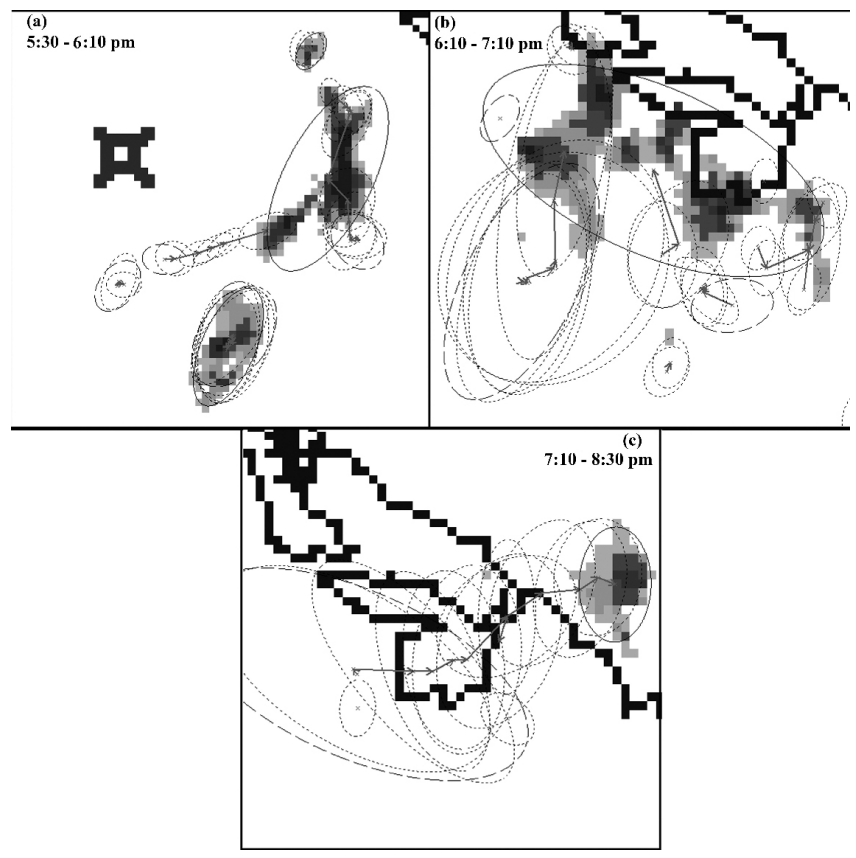

Figura 6 - Ampliación del sistema tormentoso que produjo la granizada.

En (b) de la misma figura puede verse que la gran tormenta, formada en el intervalo anterior, se mueve hacia el $\mathrm{N}$ para terminar, a las 7:10 pm, siendo parte de una de mucho mayor tamaño. En esta nueva tormenta mayor también han contribuido otras de cierto tamaño surgidas al E, incluyendo una formada en su región más oriental por la unión de tres celdas pequeñas. Han ocurrido además varias divisiones y posteriores uniones antes del instante final. El intervalo visualizado en (b) termina minutos antes del paso de la tormenta por encima de Nuevitas. A partir de las 7:10 pm la tormenta permanece sin uniones ni divisiones, como se observa en (c), pasando de esta forma sobre Nuevitas donde produjo granizos. Nótese el movimiento hacia el ENE durante todo el intervalo. 


\section{CONCLUSIONES Y RECOMENDACIONES}

El método propuesto para identificar y seguir tormentas en dos dimensiones mostró las siguientes ventajas:

- Gran habilidad en los casos simple (sin uniones/ divisiones) y moderadamente complejo (algunas uniones/ divisiones), incluyendo el caso de una tormenta tornádica ocurrida sobre Camagüey.

- Incluso para un caso de gran complejidad (múltiples uniones/divisiones), al aumentar el umbral de reflectividad para la identificación a $30 \mathrm{dBZ}$, logró seguir de manera satisfactoria una tormenta productora de granizos durante 3 horas.

- $\quad$ Simplicidad.

- $\quad$ Tiempo de corrida menor a los $10 \mathrm{~s}$ para todos los casos, en un microprocesador PIV.

Algunas de sus desventajas fueron:

Identificación en dos dimensiones, perdiendo toda la valiosa información tridimensional sobre la estructura de la tormenta.

- Un solo umbral de reflectividad para la identificación, lo cual disminuye su habilidad para detectar pequeñas celdas embebidas en grandes regiones de fuerte precipitación, como pueden ser líneas de tormentas.

- $\quad$ Método de seguimiento demasiado simple.

Algunas recomendaciones son:

Probar el método en otros casos de estudio de interés, para lo cual es ideal contar con datos de radar de alta calidad en días con reportes de severidad.

- $\quad$ Emplear múltiples umbrales de reflectividad para la identificación.

- $\quad$ Emplear la información de todos los CAPPI para obtener la estructura tridimensional de las tormentas.
- $\quad$ Incluir esta información en el seguimiento.

- $\quad$ Proponer un método de pronóstico inmediato que emplee todo lo anterior.

\section{BIBLIOGRAFÍA}

Dixon, M.; Wiener, G. TITAN: Thunderstorm identification, tracking, analysis, and nowcasting. A radar-based methodology. Journal of the Atmospheric and Oceanic Technology, v. 10, n. 6, p. 785-797, 1993.

Handwerker, J. Cell tracking with TRACE3D - a new algorithm. Atmospheric Research, v. 61, p. 15-34, 2002.

Houze, R. A. Jr. Cloud Dynamics. Ed. Academic Press, 1993, $573 \mathrm{p}$.

Johnson, J. T.; MacKeen, P. L.; Witt, A.; Mitchell, E. D.; Stumpf, G. J.; Eilts, M. D.; Thomas, K. W. The storm cell identification and tracking algorithm: an enhanced WRS88D algorithm. Weather and Forecasting, v. 13, p. 263-276, 1998.

Novo, S.; Martínez, D.; Gamboa, F. Estructura interna de diferentes tipos de tormentas convectivas observadas sobre Camagüey, a partir de datos de radar. En: X Congreso Latinoamericano e Ibérico de Meteorología, La Habana, 2003.

Novo, S.; Martínez, D.; Gamboa, F. Estudio por radar de dos tormentas convectivas observadas sobre Camagüey. En: Convención Trópico, La Habana, 2004.

Pitas, I. Digital image processing: algorithms and applications. Ed. John Wiley \& Songs, 2000. 419 p.

Pozas, W. Vesta Process 5.3.4.5. Laboratorio de Desarrollo Técnico del Centro Meteorológico de Camagüey, Cuba. 2005.

Wilson, J. W.; Crook, N. A.; Mueller, C. K.; Sun, J.; Dixon, M. Nowcasting thunderstorms: a status report. Bulletin of the American Meteorological Society, v. 79, p. 2079-2099, 1998. 